\title{
OPTICAL OBSERVATIONS OF THE BETA PICTORIS DISK
}

\author{
B. A. Smith \\ Institute for Astronomy \\ University of Hawaii \\ Honolulu, HI 96822 USA
}

Multispectral photometric and polarimetric observations of the Beta Pictoris circumstellar disk, made with a coronagraphic CCD camera at the Las Campanas Observatory between 1984 and 1989 (Smith et al 1992a), indicate that the nearly edge-on, highly flattened disk extends to a distance of more than 60 arcsec (1000au) from the star, with a nearly constant projected thickness of $2.8 \operatorname{arcsec}(45 \mathrm{au})$ on the plane of the sky. A peculiar morphological property of the disk is its apparent asymmetry, with the northeast extension appearing somewhat longer and brighter than its southwest counterpart. Because of its implications for disk dynamics and morphology, this anomalous structure is perhaps the most puzzling phenomenon yet associated with the Beta Pictoris disk and deserves further study.

The disk is composed primarily of very small particles that have nearly neutral spectral reflectivity. The light scattered by the disk has a polarization of 0.13 to 0.20 in green light, and there appears to be little or no wavelength dependence in the polarization. This suggests that the source of the polarization is Fresnel reflection from particles having very low albedo and sizes significantly larger than visual wavelengths. However, additional constraints imposed by their infrared emission require that most of the disk particles be not much larger than a few microns. An empirical relationship between polarization and reflectivity indicates that the albedo of the disk particles is 0.06 or less (Dollfus and Bowell 1971).

Thus, the disk particles may well be similar in composition to the dark, organically rich material found in meteorites, comets and certain asteroids in our own solar system. The albedo of the nucleus of Comet Halley, for example, was found to be 0.04 or less (Sagdeev et al. 1986, Keller et al. 1986). The early infall of such materials on Earth has been suggested as the principal source for the prebiotic chemical evolution that eventually led to the existence of life (Oro 1961, Chyba et al. 1990 and ref.). Thus, if similar materials are extant in the Beta Pictoris system, their presence may represent one more datum applicable to the question of life existing elsewhere in the universe.

A search for other disks of comparable areal density and extent has been carried out among more than 100 nearby dwarfs and subgiants, most within $100 \mathrm{pc}$. The negative results indicate that Beta Pictoris is abnormal, with the star surrounded by an unusually large amount of optically scattering material.

References.

Chyba, C. F., et al.: 1990, Nature 249, 366

Dollfus, A., Bowell, E.: 1971, Astron. Astrophys. 10, 29

Keller, H. U.: 1986 Nature 321, 320

Oro, J.: 1961, Nature 190, 389

Sagdeev, R. Z., et al.: 1986, Nature 321, 262

Smith, B. A., et al.: 1991a, submitted to Astron. Astrophys.

Smith, B. A., et al : 1991b, submitted to Astron. Astrophys. 